\title{
Phosphoproteomics Reveals Novel Targets and Phosphoprotein Networks in Cell Cycle Mediated by Dsk1 Kinase
}

Mei Wu ${ }^{1 \#}$, Gang Feng ${ }^{2 \#}$, Buyu Zhang ${ }^{1 \#}$, Kaikun $\mathrm{Xu}^{3}$, Zhen Wang ${ }^{1}$, Sen Cheng ${ }^{1}$, Cheng Chang ${ }^{3}$, Aditi Vyas ${ }^{4}$, Zhaohua Tang ${ }^{4 *}$, Xiaoyun $\mathrm{Liu}^{5 *}$

${ }^{1}$ Institute of Analytical Chemistry and Synthetic and Functional Biomolecules Center, College of Chemistry and Molecular Engineering, Peking University, Beijing 100871, China

${ }^{2}$ Key Laboratory of Gastrointestinal Cancer (Fujian Medical University), Ministry of Education, Fuzhou 350122, China

${ }^{3}$ State Key Laboratory of Proteomics, Beijing Proteome Research Center, Beijing Institute of Lifeomics, National Center for Protein Sciences (Beijing), Beijing 102206, P.R. China

${ }^{4}$ W.M. Keck Science Center, 925 North Mills Avenue, Claremont McKenna, Pitzer, and Scripps Colleges, Claremont, CA 91711, USA

${ }^{5}$ Department of Microbiology, School of Basic Medical Sciences, Peking University Health Science Center, Beijing 100191, China

\section{Table of Contents}

Page S-3: Supplemental Figure S1. Comparison of peptide intensities from two biological replicates of the WT samples.

Page S-4: Supplemental Figure S2. Network diagrams of physical interactions of phosphoproteins triggered by Dsk1 in different cellular processes. 
Page S-12: Supplemental Figure S3. Overexpression and purification of potential Dsk1 substrates in E. coli.

Page S-14: Supplemental Figure S4. In vitro kinase assays performed with different length of duration.

Page S-15: Supplemental Figure S5. Site-directed mutagenesis and in vitro kinase assays confirmed Ser 611 of Klp9 as the phosphorylation site.

Page S-16: Supplemental Figure S6. $d s k 1$ deletion mutants exhibited normal cell cycle progression.

Page S-17: Supplemental Figure S7. Domain organization of Klp9 and the phosphorylation site directly modified by Dsk1.

Dataset S1: Table S1. Phosphopeptide data. (Excel file)

Dataset S2: Table S2. Peptides with reduced phosphorylation levels. (Excel file)

Dataset S3: Table S3. Proteins with reduced phosphorylation levels. (Excel file)

Dataset S4: Table S4. GO Term enrichment of Dsk1 network. (Excel file)

Dataset S5: Table S5. Cell cycle protein with reduced phosphorylation levels. (Excel file)

Dataset S6: Table S6. GO Term analysis of potential Dsk1 substrates reported by Lipp et al. (Excel file)

Dataset S7: Table S7. Klp9, Chr4 and Cdr2 phosphorylation data. (Excel file) 


\section{Supplemental Figure S1. Comparison of peptide intensities from two biological replicates}

of the WT samples. The logarithmic values of peptide intensities from LC-MS measurements were plotted on each axis. The red circle contains the most data points that are located along the diagonal line, suggesting good reproducibility. The green circle contains the data points with null values in either of the two biological replicates (corresponding to those peptides detected only in one replicate). Nonetheless, those data points are still worthy of consideration, and we set a second criterion to tease out the differed phosphopeptides: their phosphorylation signals were detected at least twice in WT S. pombe cells but not in the $\Delta d s k 1$ mutant.

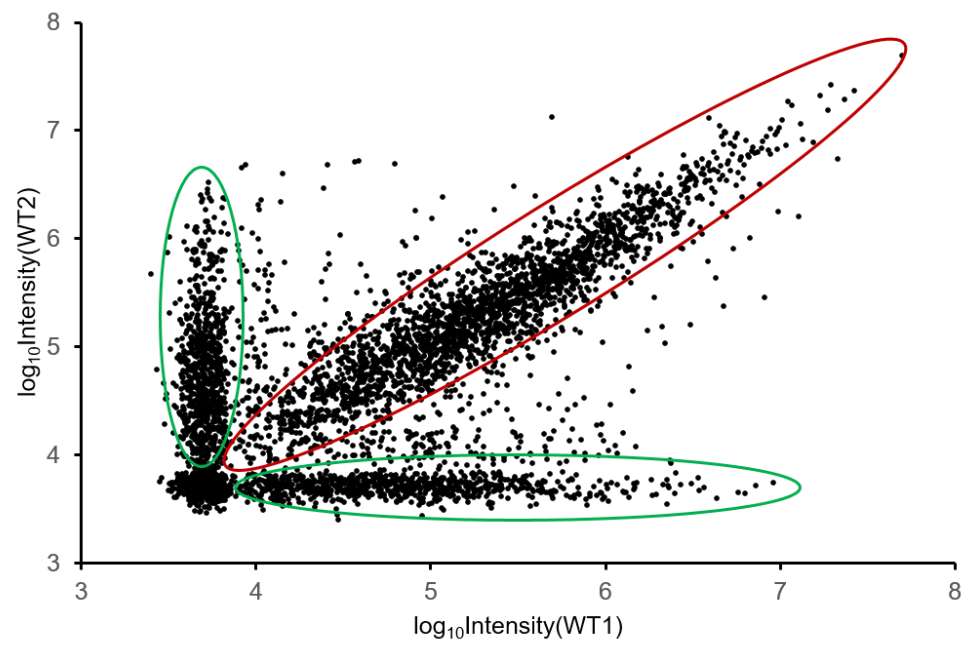


Supplemental Figure S2. Network diagrams of physical interactions of phosphoproteins triggered by Dsk1 in different cellular processes. Eight Networks A-H were assembled with espN based on the interaction data from BioGRID (PomBase.org) and displayed using Cytoscape as in Figure 3. GO Term processes are inidicated for individual networks. Node and line colors are designated similarly to Figure 3 and illustrated in (A).

A. Regulation of Meiotic Cell Cycle Phase Transition

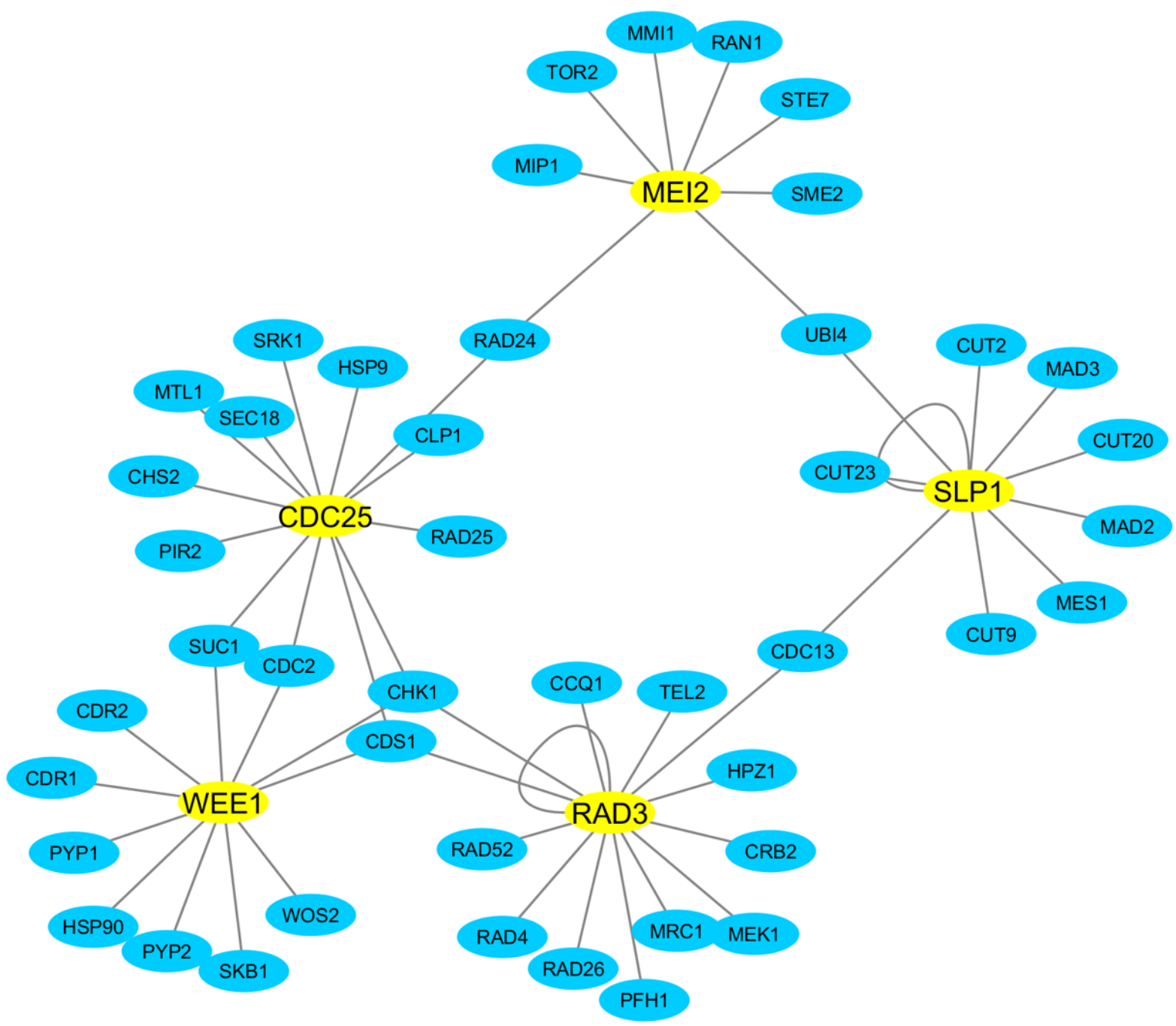

S-4 
B. Mitotic Spindle Organization

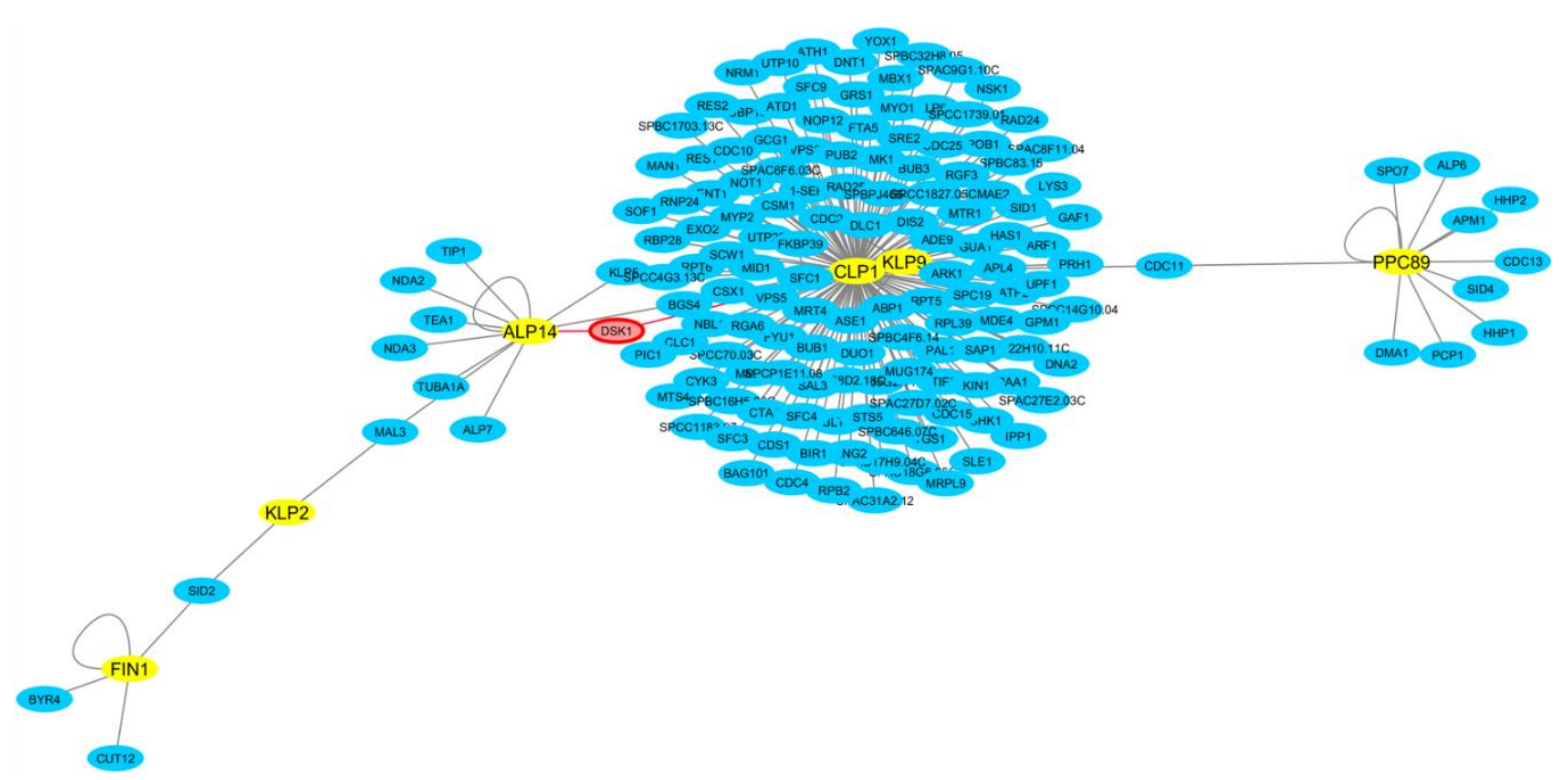


C. Cell Polarity

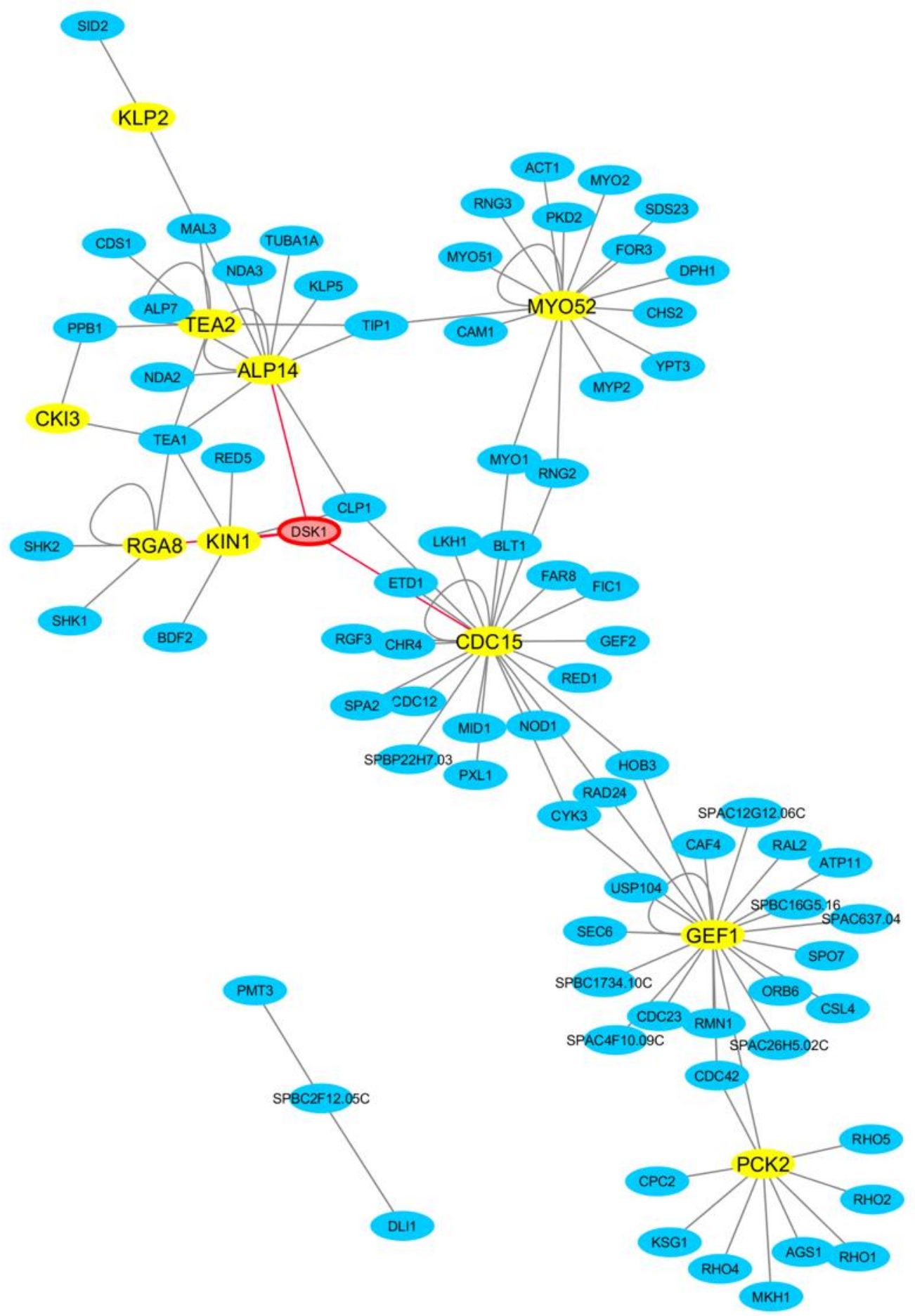

S-6 


\section{Cytoskeleton Organization}

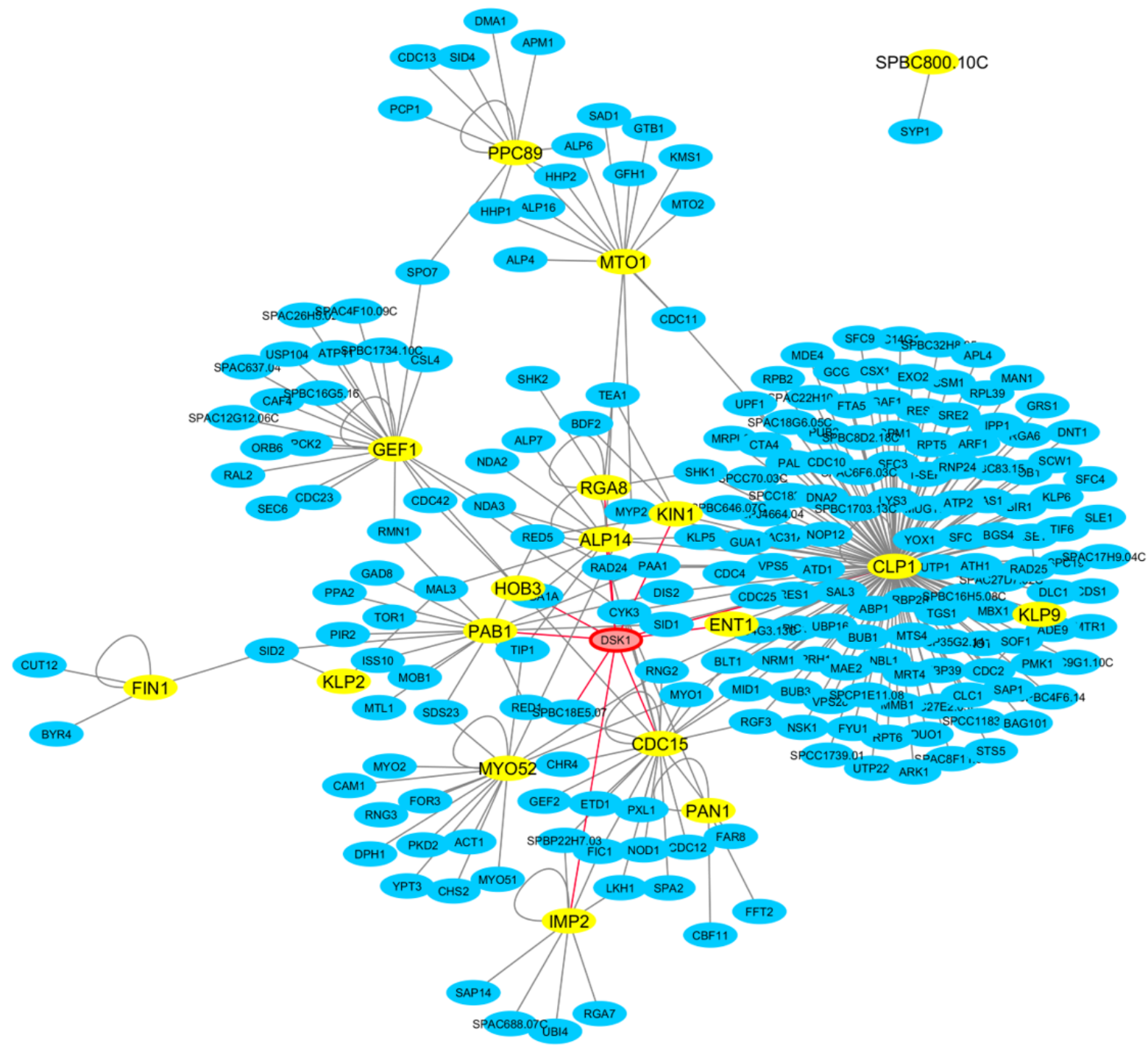




\section{E. Chromosome Localization}

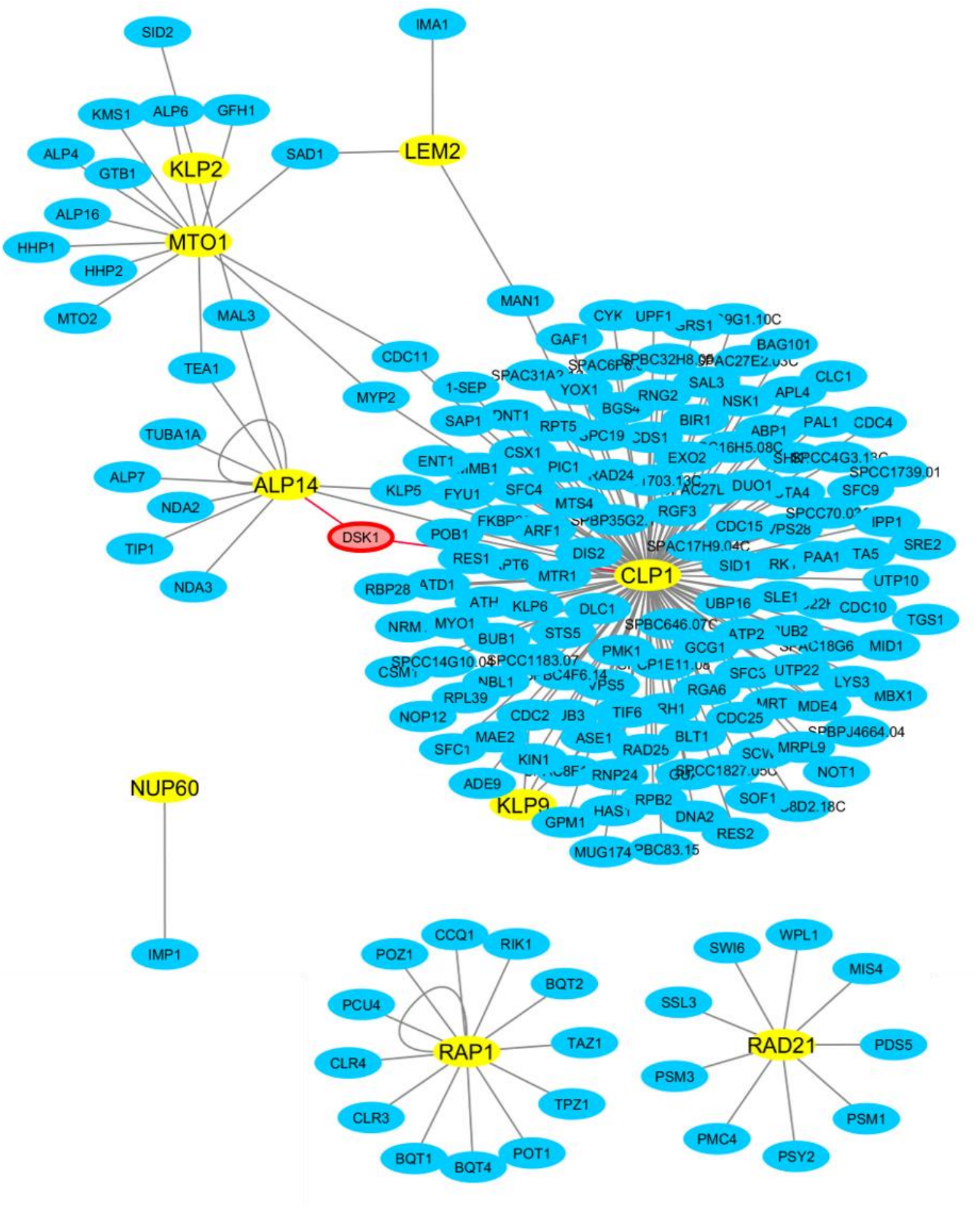




\section{F. Macromolecule Organization}

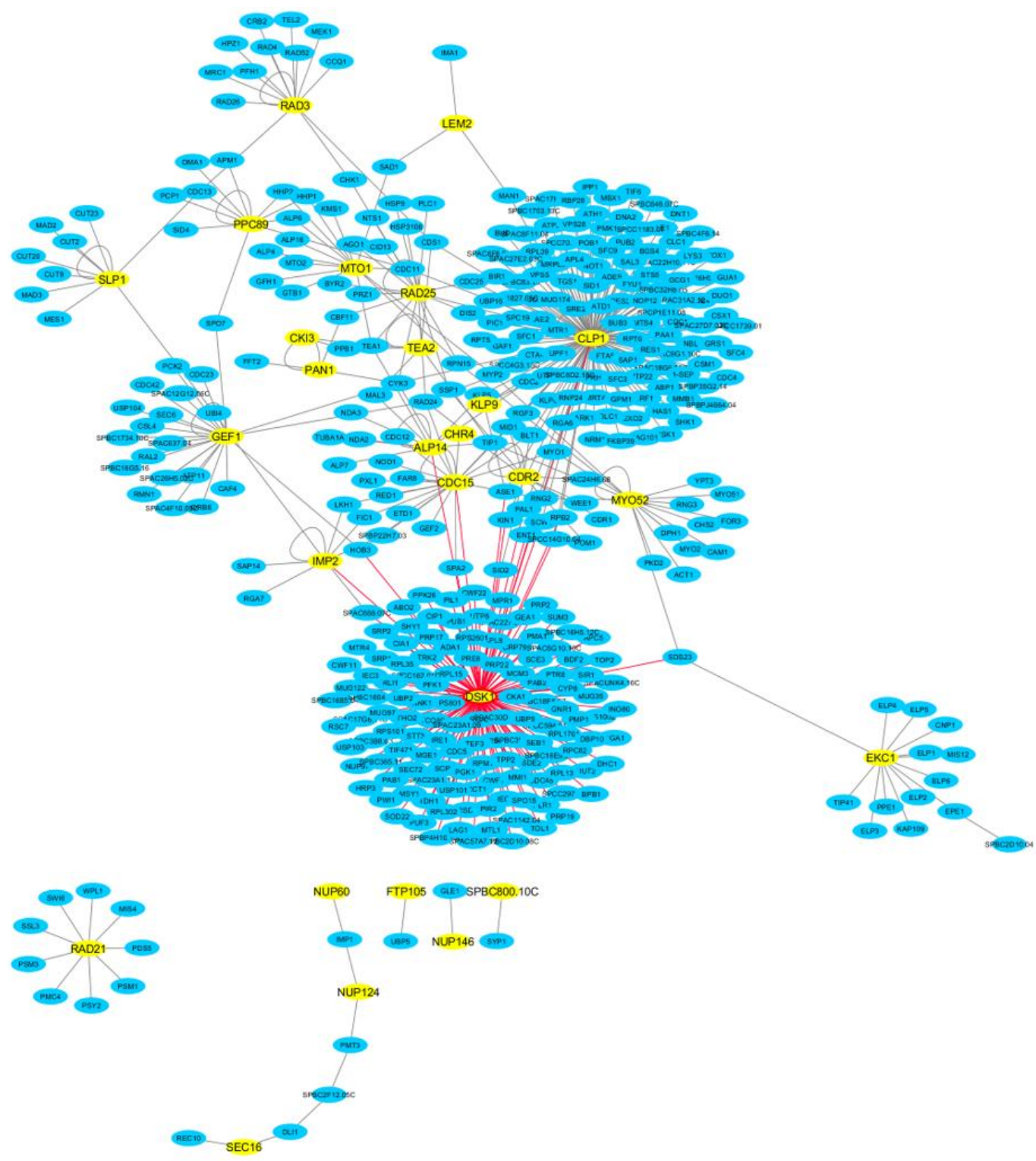




\section{G. Organelle Location}

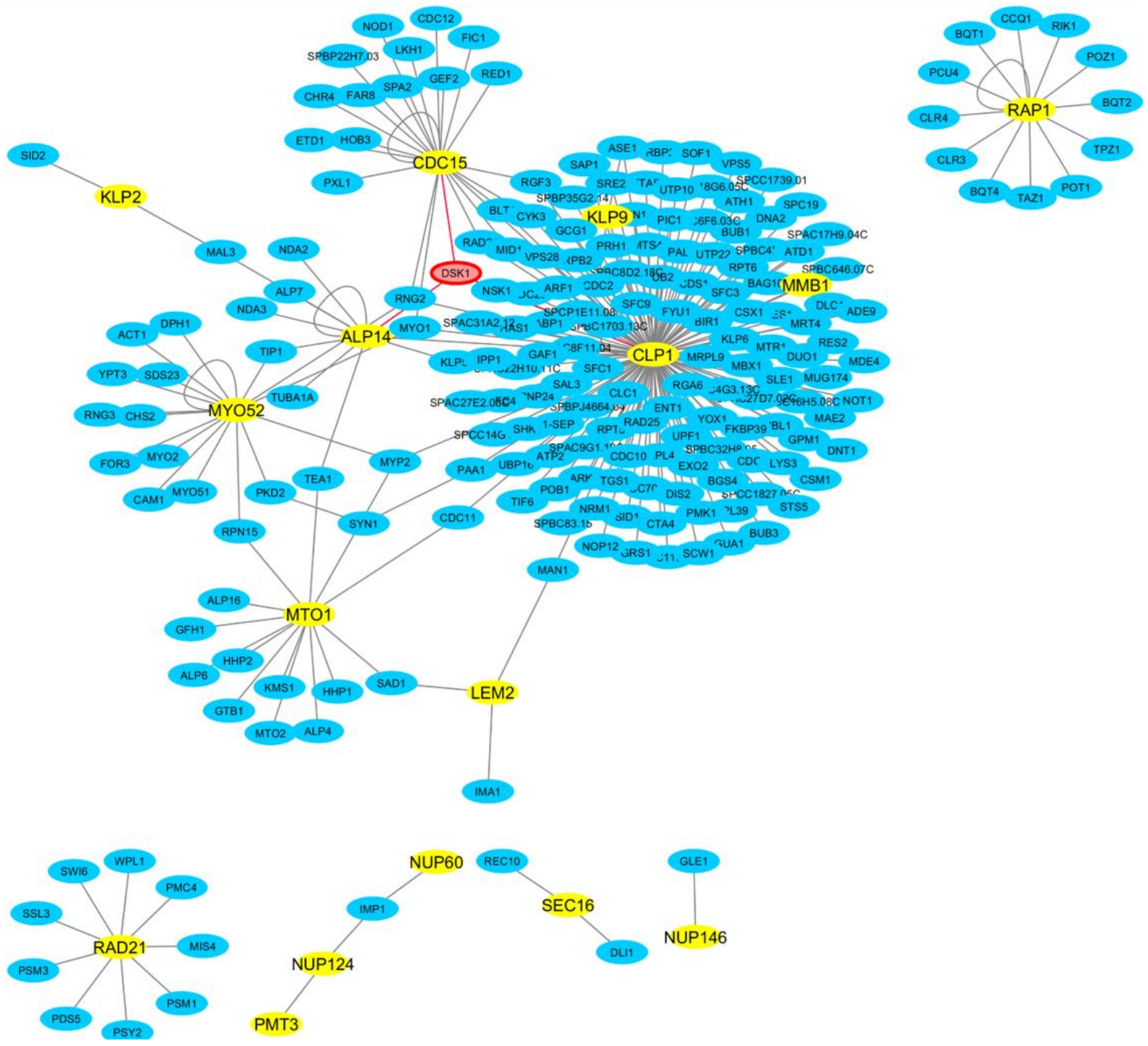




\section{H. Regulation of Biological Process}

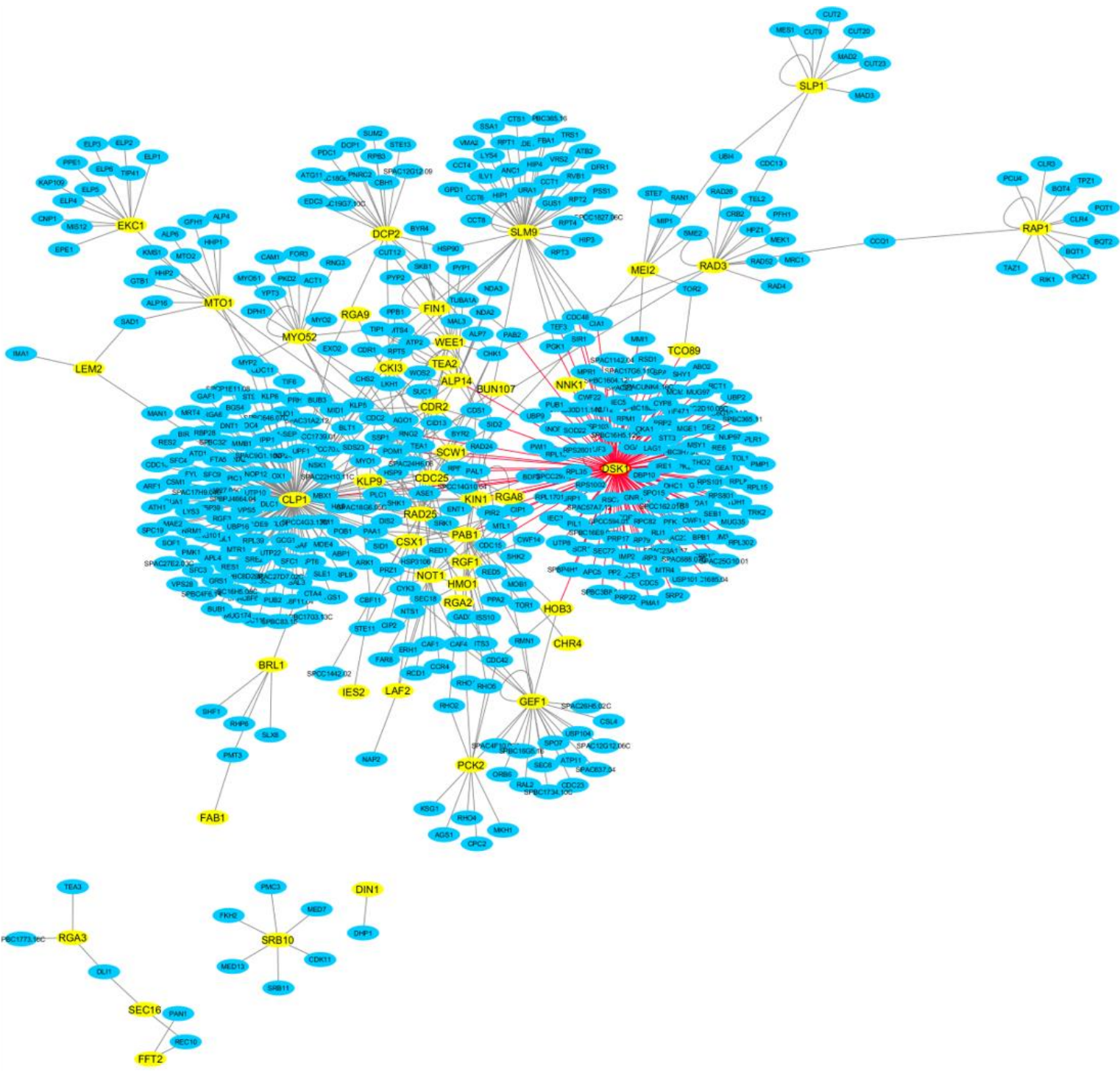




\section{Supplemental Figure S3. Overexpression and purification of potential Dsk1 substrates in}

E. coli. BL21 bacterial cells harboring plasmids with subcloned genes encoding proteins of interest were grown in LB without or with IPTG to induce the expression of His- or GSTtagged fusion proteins from pET28a-His or pGEX-6P-1-GST, respectively. After sonication, cell lysates were separated into the supernatant (containing soluble proteins) and pellet fractions (containing insoluble proteins) by centrifugation. Samples were analyzed by SDSPAGE. Lane 1, total lysate -IPTG; lane 2, total lysate + IPTG; lane 3, supernatant +IPTG; lane 4, pellet +IPTG. (A) Expression of His-tagged fusion proteins. (B) Expression of GST-tagged fusion proteins.
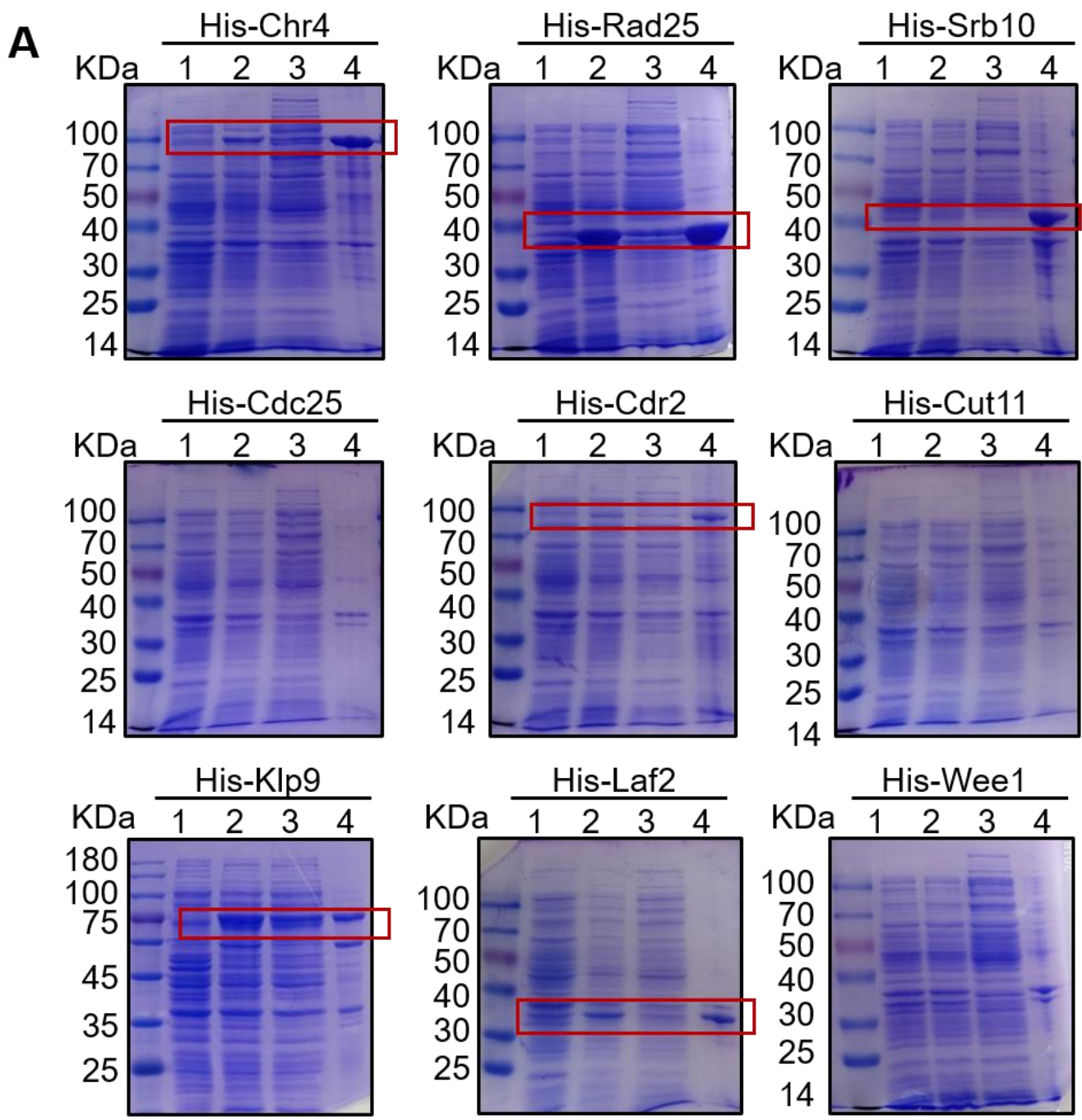

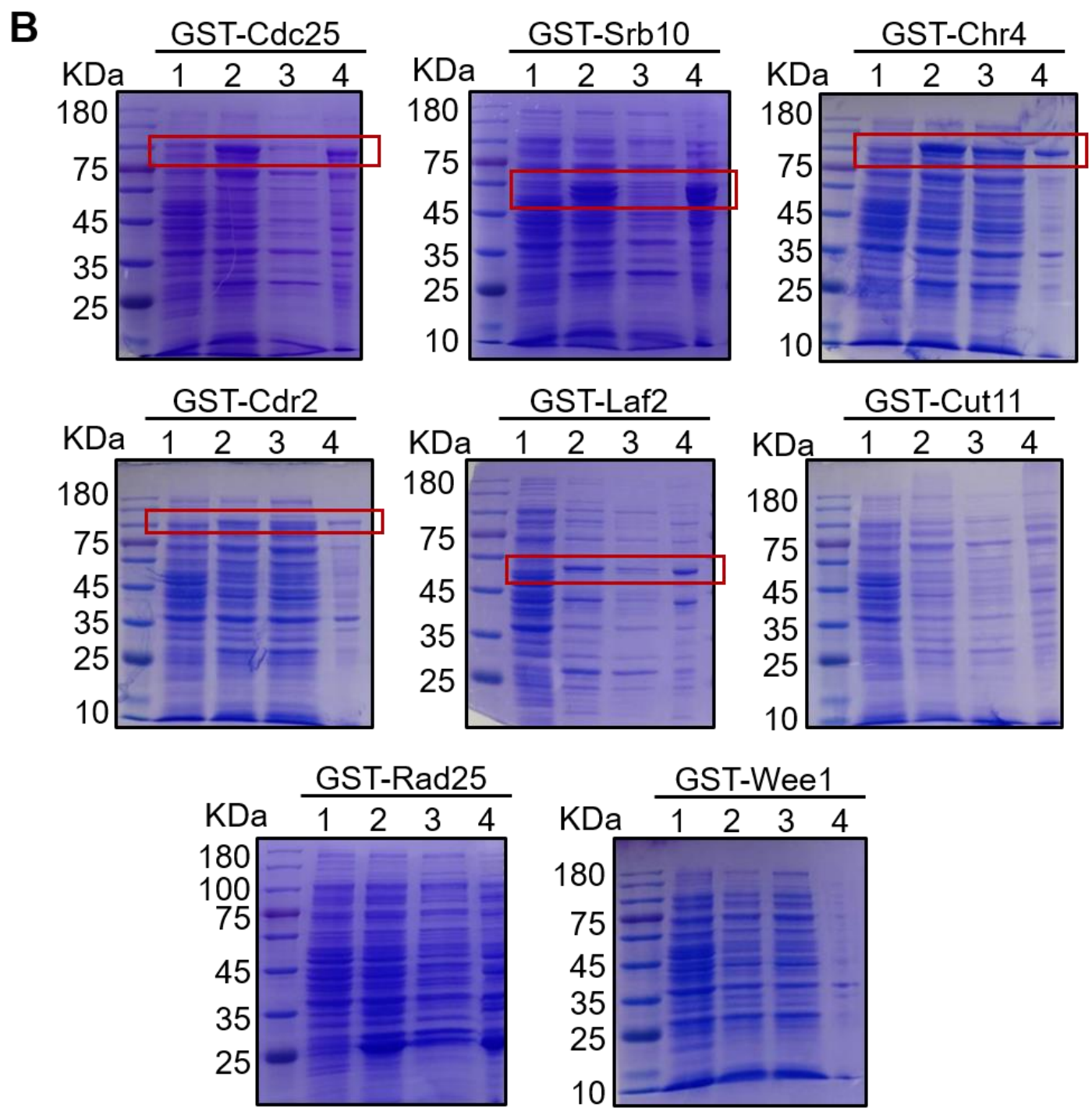
Supplemental Figure S4. In vitro kinase assays performed with different length of duration. Purified GST-Dsk1 (60 ng) and His-Klp9-WT $(2 \mu \mathrm{g})$ were incubated in a reaction buffer (50 mM Tris-HCl, pH 7.5, $150 \mathrm{mM} \mathrm{NaCl}, 10 \mathrm{mM} \mathrm{MgCl} 2,1 \mathrm{mM}$ DTT and $1 \mathrm{mM}$ ATP) for different durations at room temperature. Extracted ion chromatograms of Klp9 phosphorylated peptide $-\mathrm{V}_{603} \mathrm{VSPIKPLSPSR}_{614^{-}}$(with $\mathrm{m} / \mathrm{z}=680.78$ ) were shown to indicate the relative levels of phosphorylation.

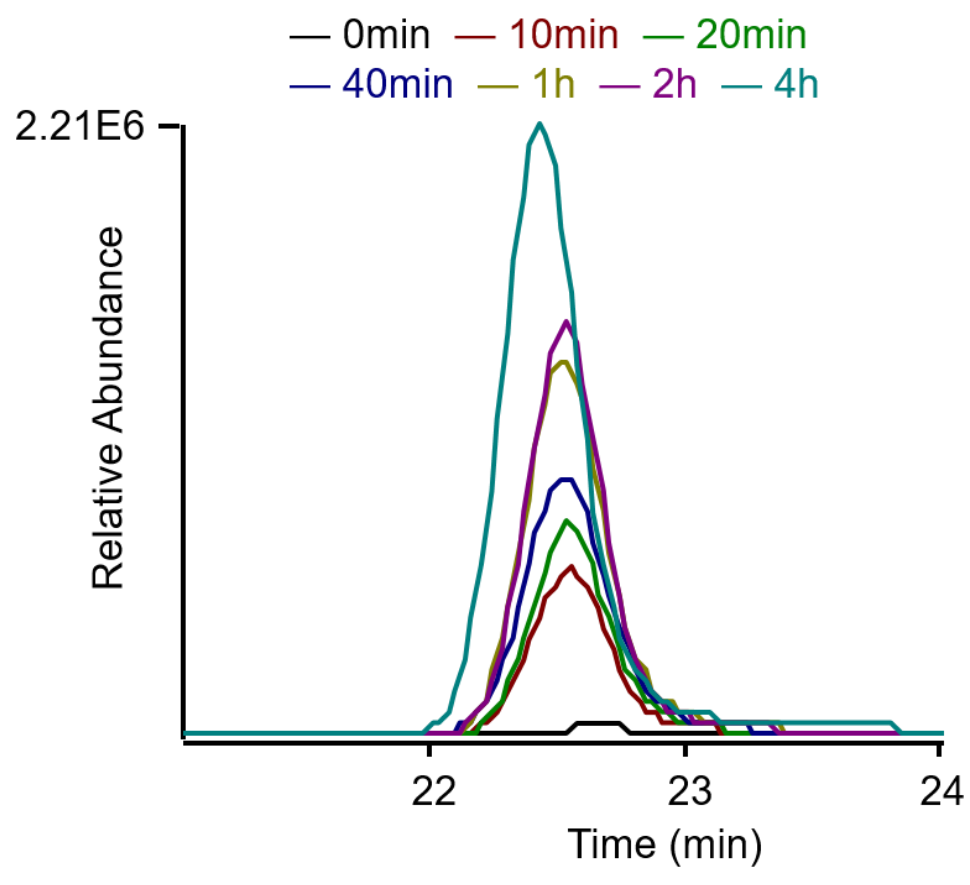


Supplemental Figure S5. Site-directed mutagenesis and in vitro kinase assays confirmed

Ser611 of Klp9 as the phosphorylation site. In vitro kinase assays with purified Dsk1, Klp9 $9_{\mathrm{S} 611 \mathrm{~A}}$, or Klp9 $9_{\mathrm{S} 613 \mathrm{~A}}$ were performed. Extracted ion chromatograms show the intensities of the non-phosphorylted (left) and phosphorylated peptides (right). (A) Mutant Klp9 $9_{\mathrm{S} 611 \mathrm{~A}}$, nonphosphorylted peptide $-\mathrm{V}_{603} V_{\text {VSPIKPLAPSR }}{ }_{14}-(\mathrm{m} / \mathrm{z}=422.2)$ and phosphorylated peptide $\mathrm{K}_{601}$ KVVSPIKPLAPSR $_{614-}(\mathrm{m} / \mathrm{z}=801.0)$. (B) Mutant Klp9 ${ }_{\mathrm{S} 613 \mathrm{~A}}$, non-phosphorylted peptide $\mathrm{V}_{603}$ VSPIKPLSPAR $_{614-}(\mathrm{m} / \mathrm{z}=632.8)$ and phosphorylated peptide $-\mathrm{K}_{601} \mathrm{KVVSPIKPLSPAR}_{614-}$ $(m / z=801.0)$.

A

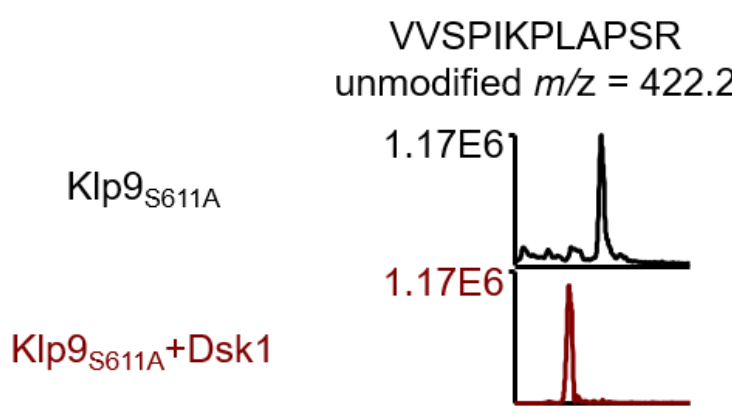

B

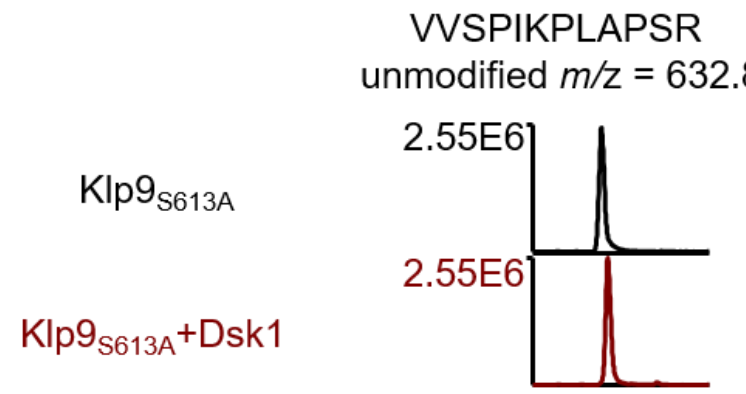

KKVVSPIKPLSPAR modified $\mathrm{m} / \mathrm{z}=801.0$

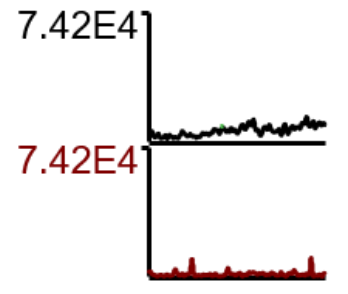

KKVVSPIKPLSPAR modified $\mathrm{m} / \mathrm{z}=801.0$

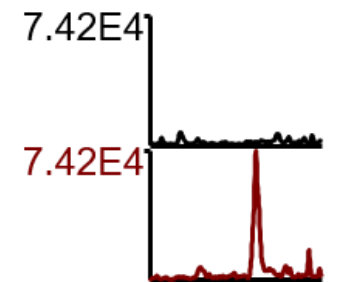


Supplemental Figure S6. $d s k 1$ deletion mutants exhibited normal cell cycle progression.

(A) Two independent $d s k 1$ deletion mutants showed growth curves similar to that of WT cells.

(B) FACS experiments demonstrated normal cell cycle progression of $d s k 1$ deletion mutants indistinguishable from that of WT cells after HU synchronization to S phase.

A

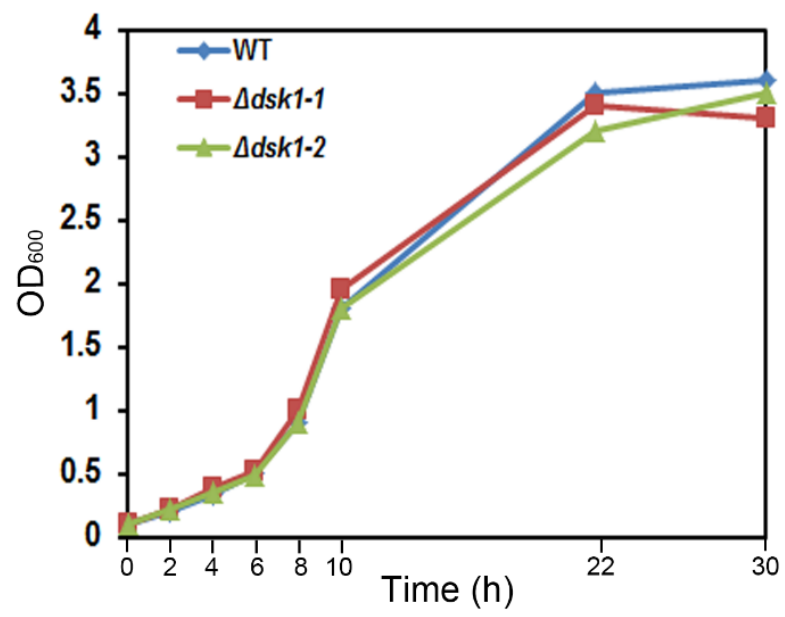

B

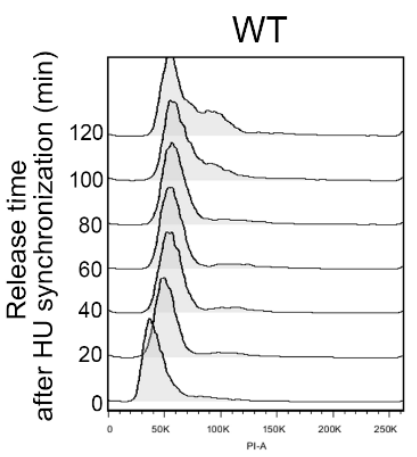

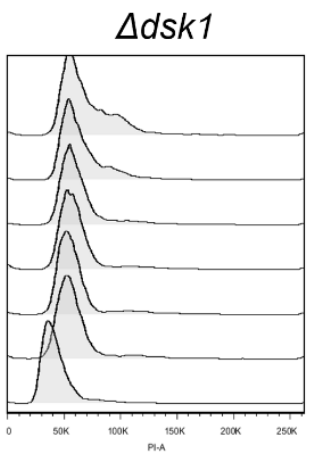


Supplemental Figure S7. Domain organization of Klp9 and the phosphorylation site directly modified by Dsk1. Klp9 domain arrangements in which kinesin motor (green bar), coiled coil (green boxes), low complexity (blue), and disorder (gray) domains are schematically presented (http://pfam.xfam.org/protein/Q1MTQ7). Residues 546-578 contain a putative NLS 47. Three known phosphorylation sites in the C-terminal tail of low complexity domain (amino acid residues 604-622) are indicated. Ser 611 is confirmed as the phosphorylation site by Dsk1 in this study.

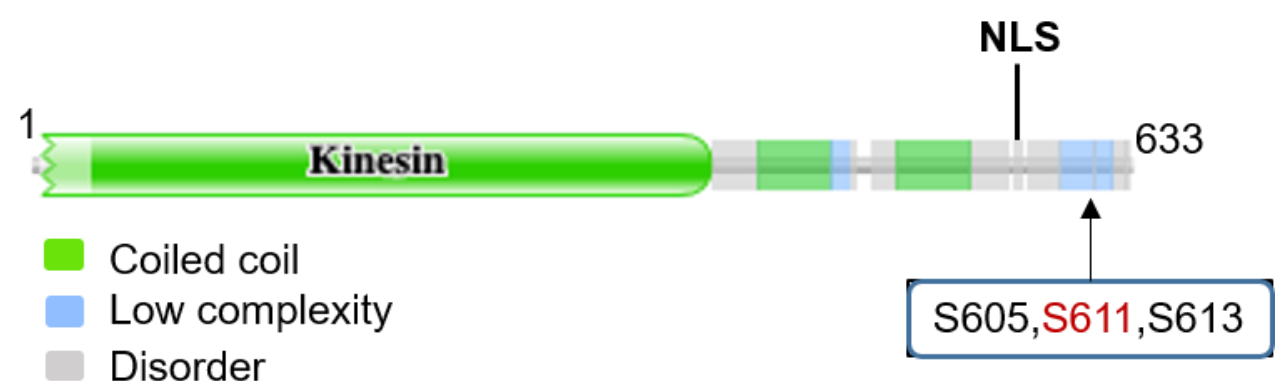

